\title{
Modelli strutturali del cantare "fiabesco" italiano
}

È noto che la produzione di cantari dei secoli XIV e XV si caratterizza per la sua estrema varietà dal poemetto narrativo del tipo Fiorio $e$ Biancifiore, alla composizione sermoneggiante dell'Atrovare del vivo e del morto, alla novella borghese, tipo La storia del calonaco di Siena, al frammento di letteratura romanzesca, quale i Cantari di Tristano. È noto anche che uno dei motivi di maggior interesse nell'attenzione che la ricerca contemporanea dedica ai cantari tre-quattrocenteschi sta nel loro essere documento di "letteratura popolareggiante," a metà strada dunque fra la produzione "colta" delle avanguardie socioculturali, conscia della sua letterarietà, e il fenomeno sociale dell'intrattenimento popolare, fenomeno a cui appartiene la tradizione narrativa del folclore: fiabe, canzoni, leggende e così via. ${ }^{1}$ Sembra dunque che uno studio ravvicinato dei cantari possa avere un interesse anche teorico nella misura in cui servirà a rivelare le modalità delle interrelazioni fra "letteratura colta" e "memoria popolare" nei due aspetti fondamentali di a) fluttuazione e/o passaggio di motivi dalla tradizione popolare orale all'elaborazione poetica scritta o viceversa, a livello di contenuti e $b$ ) contaminazione di strutture narrative.

Il presente articolo si occupa esclusivamente dell'aspetto strutturale del cantare italiano cosiddetto fiabesco, rimandando a un articolo successivo lo studio dei motivi. Abbiamo scelto di affrontare la questione partendo dallo studio dei "cantari fiabeschi" per queste ragioni: la ricerca di un modello formale, come quello magistralmente enucleato da V. Propp per la fiaba classica di magia, appare perfettamente adeguato allo studio di una produzione largamente ripetuta nel tempo e nello spazio quale, appunto, la fiaba magica o il mito nel dominio del folclore, gli epigoni della letteratura cavalleresca nel nostro medioevo e nel Rinascimento, e probabilmente la letteratura di massa dei tempi odierni. Perché il modello formale, o schema narrativo, è fondamentalmente un formidabile strumento mnemonico, è ciò che permette al genere di conservarsi, e ai contenuti che si vogliono trasmettere di essere 
facilmente recepiti e tramandati. Meno adeguata appare la ricerca strutturale di tipo proppiano alla letteratura "colta," profondamente individuale e che, di solito, si affida alla novità dell'idea, dell'immagine, della forma, anziché inserirsi consenziente in un alveo tradizionale determinato. Ora, molti dei nostri cantari sono rielaborazioni a destinazione canterina di opere letterarie, sia pure consacrate da uno straordinario successo e da una straordinaria diffusione, come il romanzo di Tristano, o i Vangeli, o le novelle del Boccaccio, e perciò stesso debbono avere conservato in misura rilevante i caratteri propri di quell'opera letteraria, al di là naturalmente delle trasformazioni superficiali dovute alla forma (l'ottava rima) e alle esigenze di una recitazione pubblica. Se si vuole andare alla ricerca di un modello formalizzante per il cantare in quanto opera destinata ad una produzione - per così dire - di massa, e dunque ad inserirsi consapevolmente in una tradizione di forme, di temi e di motivi pre-determinati, contemporaneamente consolidandola e modificandola, e ad essere recepita con favore da un largo pubblico, bisognerà andare in cerca di quei cantari che offrono un più largo margine di autonomia dalle fonti dalle quali accolgono, com'è ovvio, tutta una rete di motivi, ma integrandoli in un intreccio di cui l'autore canterino sia il solo responsabile e corrisponda alle precise esigenze funzionali del mestiere. In questo modo, io credo, si potranno cogliere i criteri che hanno inconsciamente guidato il canterino, o chi per lui, a comporre la sua bella favola, e si potrà rintracciare uno schema narrativo valido, se non altro, per un certo numero di cantari.

Il secondo requisito per la scelta del corpus è stato la brevità. Giustamente P. Zumthor ha sottolineato nel suo Essai de poétique médiévale ${ }^{2}$ la differenza radicale che passa fra la struttura della poesia epica e del romanzo, e le realizzazioni di quella che egli chiama "la durée close," comprendente manifestazioni in apparenza diversissime come i poemetti, del tipo La Châtelaine de Vergi, i lais, i fabliaux, le leggende sacre ecc. Per quanto riguarda il cantare trecentesco e quattrocentesco italiano, invece, probabilmente a causa della prospettiva che lo ha considerato per lungo tempo solo come una piattaforma preparatoria per i grandi poemi del Pulci, del Boiardo e dell'Ariosto, si è teso finora a non fare distinzioni e a considerarlo parte di quella produzione cavalleresca che comprende normalmente anche il lungo poema o il romanzo in prosa, in ciò aiutati naturalmente dalla comunanza del metro, delle formule e, in parte, del contenuto. É necessario invece, a mio parere, non dimenticare che il cantare breve è l'erede e il corrispondente italiano della tradizione della Kurzerzählung francese. 
I cantari che abbiamo scelto a costituire il nostro corpus sono dunque :

1. Il Bel Gherardino, ${ }^{3}$ in due canti (736 versi). Trascritto intorno al 1370 , sembra uno dei più antichi cantari del genere a cui abbiamo accennato. È nota la testimonianza del Boccaccio che così stigmatizza la vedova del Corbaccio: "E già assai volte, millantandosi, ha detto che se uomo stata fosse, l'arebbe dato il cuore d'avanzare di fortezza non che Marco Bello, ma il Bel Gherardino che combattea con l'orso." ${ }^{\prime 4} \mathrm{Ma}$ anche la terza novella della seconda giornata del Decameron (completato prima della stesura del Corbaccio) sembra aver tratto ispirazione da una narrazione corrispondente alla prima parte del Bel Gherardino, e che avrebbe già assunto alcuni tratti specifici di una elaborazione italiana, distinta da quella originaria francese.

2. Liombruno, ${ }^{5}$ in due canti (776 versi). Com'è noto, di questo cantare non esistono testimonianze manoscritte, all'infuori di un manoscritto miscellaneo della Biblioteca Nazionale di Parigi (Fonds Ital.1095), che riporta due frammenti di una redazione diversa dalla vulgata, trascritti nel $1474 .^{\circ}$

Ai due versi già segnalati dal Levi come identici nel Liombruno e nel Bel Gherardino ${ }^{7}$ se ne può aggiungere ora un terzo, documentato dalla seconda redazione del Liombruno: avvicinandosi a Liombruno fanciullo, l'aquila che lo porterà con sé

... disse: "Levate su dritto

io son colei che te farrò contento." (vv. 85-86)

così come la Fata Bianca, entrando all'improvviso nel letto del Bel Gherardino lo rassicura:

..."Non avere di me pavento,

ch'io sono colei che.tti farà contento." $(I, 23,7-8)$

Questo sia detto non tanto per rivendicare, come faceva il Levi, l'antichità del Liombruno, quanto per introdurre l'ipotesi che il cantare di Liombruno quale noi lo conosciamo non solo sia posteriore al Bel Gherardino, ma lo abbia utilizzato come fonte (insieme, ovviamente, ad altre); confermerebbe il fatto anche il motivo del torneo che, collocato, come nel Bel Gherardino, al culmine della ricerca del bene perduto, e coronato dal ricongiungimento con la Fata Bianca, sembra perfettamente integrato nella struttura narrativa del cantare, mentre nel Liombruno, posto com'è a interrompere senza ragione il ritorno di Liombruno da Aquilina, sembra rivelare il cedimento dell'autore del cantare a una suggestione estranea allo 
svolgimento lineare della leggenda. Tanto più che compare nella giostra del Liombruno anche

... un saracin tanto possente

che della giostra quasi era vincente (I, v. 264)

e che ricorda tanto da vicino il Soldano, campione della giostra, la cui presenza, di nuovo, è meglio giustificata nel Bel Gherardino poiché l'eroe è stato suo prigioniero e ha promesso di ucciderlo alla moglie di lui.

3. La Ponzela Gaia, ${ }^{8}$ senza suddivisioni( 864 versi). Giustamente il Varanini rileva, sulle orme del Levi, una notevole vicinanza di questo cantare alle versioni francesi (Lanval e Graelent) nella prima parte, e alle vicende narrate nella Tavola Rotonda nella seconda parte. Documenta anche l'esistenza di un testo in prosa toscana (il cantare, come si sa, è stato restituito alla veste linguistica dialettale del codice quattrocentesco che lo ha conservato) "il cui contenuto doveva almeno in parte coincidere con la vicenda del nostro poemetto," e di cui è rimasto soltanto il frammento finale. Supponendo che questa narrazione in prosa fosse nota all'autore del cantare, la conoscenza dell'intero testo avrebbe certamente permesso delle osservazioni interessanti sulle trasformazioni strutturali subite dal racconto nel passaggio da un testo presumibilmente strutturato secondo le modèle romanesque, nonostante la relativa brevità del racconto, ${ }^{9}$ alla narrazione tipo cantare. Si può comunque, già dal confronto col solo frammento, osservare la molto maggiore rapidità della conclusione nel cantare: alla liberazione della Ponzela Gaia e alla punizione delle sue persecutrici, seguono immediatamente il ritorno alla corte di re Artù e i festeggiamenti per i due amanti, salvo una brevissima sosta al castello di una dama fedele alla Ponzela Gaia, che è la ripresa e la conclusione di un motivo incontrato nel corso della narrazione precedente; il frammento in prosa fa scontrare Galvano con dodici cavalieri sconosciuti che vengono dall'eroe vinti, acciecati, e spediti alla corte di Artù a preannunciare il suo arrivo: l'andamento del racconto sembra dunque inserirsi in quel modello di sequela ininterrotta, e per lo più assolutamente immotivata, di incontri scontri e avventure che, una volta perduto il senso della finissima tecnica francese dell'entrelacement, rimane la caratteristica delle compilazioni romanzesche italiane di materia arturiana. Varanini stesso osserva invece che il cantare "ha una struttura narrativa organica e salda"; maggiori precisazioni riguardo a questa struttura saranno il risultato dell'analisi che stiamo per intraprendere.

4. Gismirante, ${ }^{10}$ in due canti ( 848 versi). Il cantare, conservato in 
un manoscritto quattrocentesco, è sicuramente attribuibile al Pucci, che così si firma nell'ultimo verso:

Al vostro onor questo fe' Antonio Pucci.

Afferma il Levi: "La novella certamente non è stata inventata dal Pucci, prima di tutto perché queste fiabe non si inventano mai, e poi perché il poeta stesso ci avverte che egli ne ha tratto l'argomento da un libro .... che egli andava assiduamente sfogliando per trarne piacevoli 'novità'.... Ma quale fosse il libro che il Pucci aveva sotto gli occhi, non saprei indicare con precisione." diamo a uno studio ulteriore la discussione delle fonti e dei motivi del Gismirante $e^{12}$ contentandoci in questo articolo di far risaltare l'omogeneità strutturale di questo con gli altri cantari del corpus.

5. Gibello, ${ }^{13}$ senza suddivisioni ( 696 versi), testimoniato da un codice quattrocentesco (Laur. Pal. CXIX). Él'unico che svolge le sue avventure su un piano coerentemente cavalleresco, senza alcuna intrusione magica o soprannaturaie; i tempi e l'andamento del racconto sono tuttavia così vicini a quelli degli altri cantari (risalendo per i motivi alle stesse fonti), che la sua inserzione in questo corpus non ne disturba l'omogeneità. I critici che ne procurarono l'edizione (F. Selmi, nella dispensa XXXV della "Scelta di curiosità inedite o rare)," Bologna 1863, e E. Levi) concordano nel riconoscere tracce linguistiche di una fonte francese particolarmente nei nomi delle città e dei protagonisti.

\section{FONTI E MOTIVI}

Presentando i cantari del nostro corpus non abbiamo potuto esimerci dal parlare di fonti delle nostre storie. Di fonti parlano anche esplicitamente alcuni dei cantari stessi - e le tracce dell'elaborazione di un testo seguito presumibilmente da vicino sono particolarmente evidenti in certi punti del Bel Gherardino; mentre, poco prima, indicavamo nella "relativa autonomia" dell'elaborazione uno dei requisiti per la scelta del corpus. Tuttavia, è molto importante conservare una precisa distinzione fra fonte di un motivo, e fonte di un'opera; probabilmente nessuno degli episodi che costituiscono il contenuto di questi cantari è invenzione del suo autore, ma quello che è importante, e di cui andiamo in cerca, è il modo in cui questi diversi motivi sono stati combinati fra loro. $\dot{\mathrm{E}}$, a nostro parere, l'intreccio (un certo tipo di intreccio) che costituisce uno dei tratti distintivi di questa letteratura, e contribuisce a creare il "canone" che rende ragione della scarsa individualità dei cantari, della loro "coralità." I motivi utilizzati dai nostri cantari 
sono da ricercarsi quasi esclusivamente nei lais brettoni (Lanval, Graelent, Guingamor, Désiré, Guigemar), e nel poema Partonopeus de Blois. Tuttavia, i lais brettoni - anche i rifacimenti più tardi hanno una struttura narrativa notevolmente diversa da quella che è possibile ricavare dall'esame dei nostri cantari. ${ }^{14}$ Né il lunghissimo poema Partonopeus de Blois (10856 versi) può essere stato travasato nella struttura dei cantari senza un'operazione radicale di sfoltimento e di semplificazione.

Ci sembra dunque corretto parlare di relativa autonomia di una determinata porzione della produzione in ottava rima che non intraprende la trascrizione pura e semplice di un'opera precedente, rispettandone, oltre al contenuto, il ritmo e l'architettura. Quali siano invece il ritmo e l'architettura di questa determinata porzione della produzione in ottava rima è quanto cercheremo di determinare nelle pagine seguenti, servendoci della metodologia instaurata da Propp, che in questo contesto appare perfettamente appropriata, e per la caratteristica del cantare di essere una produzione di massa, e per l'utilizzo di elementi fiabeschi che, come vedremo, vanno ben oltre la semplice funzione di motivi dell'intreccio, ma affèttano profondamente la struttura narrativa del cantare.

\section{ANALISI STRUTTURALE: MITO E FIABA}

Come già rilevato da tutti quelli che hanno avuto ad occuparsi di questi cantari, è evidente che la "storia di Lanval," con la sua concatenazione disi) situazione di mancanza $b$ ) incontro con una fata che ripara alla povertà dell'eroe e gli concede il suo amore $c$ ) interdizione di manifestare l'amante soprannaturale (tabù legato all'innaturale relazione di un mortale con una fata) $d$ ) infrazione del divieto e disgrazia del mortale, è riprodotta senza variazioni nella prima parte (quando il cantare è in due canti, coincidente col primo canto), del Bel Gherardino, del Liombruno e della Ponzela Gaia. $\mathrm{Ma}$, possiamo aggiungere, anche nel Gismirante è riprodotta, nel primo canto, una vicenda molto simile; solo che la riduzione della donna da fata a principessa comporta l'eliminazione del tabù e della relativa infrazione: la perdita della fanciulla e l'infelicità del protagonista sono invece provocate dall'intervento esterno dell' "Uomo Selvaggio."

Nella "storia di Lanval" occupa un posto importante quella che si potrebbe chiamare la "punizione" del cavaliere, con la sua lunga attesa di un supplizio, e l'agonia del rimorso di aver offeso la propria amante; il riacquisto della felicità avviene invece rapidamente, non appena la fata riappare per salvare il cavaliere da 
morte; la riconciliazione è appena appena protratta nel Graelent. Nei cantari italiani, invece, fra la perdita della donna e del suo favore e la riconciliazione, è inserita una lunga fase, che riempie in genere il secondo canto, e che assume la forma di una quête. Le modalità nelle quali la quête si realizza nei diversi cantari sono assolutamente divergenti: bisogna dunque ammettere che, almeno per la seconda parte, ciascun autore ha fatto ricorso a fonti e a motivi differenti.

Nel Gibello, come nel Gismirante, la principessa è una donna mortale, dunque anche qui la sua perdita è provocata non dall'infrazione di un tabù, ma da un intervento esterno: in questo caso, dalla rivelazione dell'oscurità dei natali dell'eroe, il che provoca una quête rivolta non direttamente al ritrovamento dei genitori: tuttavia anche nel Gibello il raggiungimento della quête è collegato al matrimonio con la principessa.

Passando dunque ad esaminare le tappe narrative attraverso le quali si snoda il racconto dei cantari, applicheremo senz'altro, in prima approssimazione, le categorie di Propp; tuttavia, poiché la struttura del cantare non si identifica con quella della fiaba, saranno necessari degli aggiustamenti e degli adattamenti dello schema proppiano, alcuni superficiali, altri più profondi. In particolare:

1. per ogni cantare, si rintracceranno gli elementi che, pur rispondendo a una determinata funzione dello schema fiabesco, assumono una configurazione tipicamente cavalleresca;

2. il nucleo mitico della "storia di Lanval" appare meglio formalizzabile con le categorie elaborate dal Dundes ${ }^{15}$ per i miti dei pellerossa americani che non con quelle della fiaba;

3. il tema stesso (cavalleresco) dei nostri cantari obbliga a condurre una ricognizione nella direzione del romanzo cavalleresco. Mi sono servita, per le osservazioni sul romanzo cavalleresco pertinenti ugualmente al cantare, della formalizzazione elaborata da A. Pasqualino sui Reali di Francia. ${ }^{16}$

Abbiamo finalmente cercato di individuare alcuni elementi specifici del cantare e di arrivare alla costruzione di uno schema strutturale che dovrebbe essere riconosciuto come uno degli elementi caratterizzanti di almeno una certa varietà del cantare trecentesco e quattrocentesco italiano.

\section{Bel Gherardino}

$\mathrm{E}^{\prime}$ costituito narrativamente da tre movimenti: uno che conduce il protagonista all'ottenimento della "felicità" - amore della fata e ricchezza; l'altro che conduce alla perdita di questa felicità; il terzo 
che conduce alla riconquista del bene perduto. Un secondo filo narrativo, relativo al compagno del protagonista, Marco Bello, si distribuisce secondo una delle schematizzazioni di Propp:

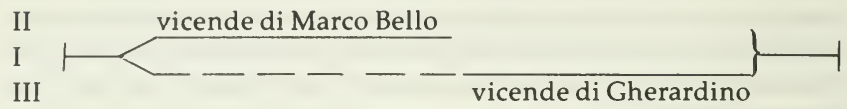

ma non si presta a una formalizzazione fiabesca.

Primo movimento

$\begin{array}{ll}\beta^{2} & \text { morte del padre }{ }^{17} \\ \gamma \delta^{1} & \text { vita cavalleresca di Gherardino } \\ a & \text { povertà } \\ 1 & \text { partenza da Roma in compagnia di Marco Bello } \\ \text { (E) } & \text { lotta con un ser ente e con un orso } \\ \mathrm{G}^{5} \mathrm{~W}^{0} & \text { castello incantato - sposa magica }\end{array}$

L'apertura del cantare - morte del padre che lascia tre figli è tipicamente fiabesca. Anche nel motivo del giovane caduto in povertà per eccesso di cortesia ci sembra di poter riconoscere una trasfigurazione cavalleresca del procedimento fiabesco che prevede il danneggiamento causato dalla trasgressione di un ordine: anche se non c'è un ordine esplicito, è evidente che il giovane Gherardino si comporta in maniera non assennata rispetto alle regole di comportamento imposte dalla società e, in questo caso, anche dalla famiglia: "e gli fratelli n'avien gran dolore/ perché facea contra al lor volere" $(1,5,5-6)$. Indico con G (Trasferimento magico nell'altro regno) il contesto del palazzo incantato, che costituisce naturalmente in questo caso un "mezzo immobile" per superare il confine fra i due regni, poiché l'atmosfera incantata del palazzo è chiara indicazione dell'alterità del mondo soprannaturale nel quale è venuto a trovarsi Gherardino.

Notiamo che Gherardino conduce con sé Marco Bello, il quale non assolve nessuna funzione narrativa necessaria - se riferito allo schema della fiaba. Notiamo anche che i due incontrano una "lotta epica," che distributivamente deve identificarsi con le prove che rendono il protagonista degno di raggiungere il suo scopo, ma senza l'intervento di alcun "donatore": benché gli animali stessi siano "fatti per incantamento," questo episodio si avvicina in realtà alle prodezze che nella tradizione delle "enfances" cavalleresche 
compie il giovane eroe a dare chiara indicazione del suo valore e di quanto ci si possa aspettare da lui.

Secondo movimento

$\S$ Decisione di tornare a Roma

$\left(\mathrm{F}^{1}\right)$ Dono di un guanto magico

$\boldsymbol{\gamma}^{1} \quad$ Interdizione

(†) Ritorno a Roma

$\delta^{1} \quad$ Trasgressione

a Perdita di ogni beneficio
$\mathrm{L}$

Int.

Viol.

Conseq.

Nessuna delle funzioni di questo movimento si identifica con un motivo tipicamente cavalleresco. Si noterà tuttavia come, messo a confronto con la griglia proppiana, la sequenza delle funzioni appare singolarmente scompigliata. Considerato in relazione alla sequenza precedente, il ritorno a casa di Gherardino corrisponde, distributivamente, alla funzione del ritorno ( $\downarrow)$, con la differenza, però, che Gherardino non porta con sé il bene acquistato, ma si allontana dalla "felicità." Il dono del guanto magico, che fornirà a Gherardino tutte le ricchezze che vuole, può far pensare alla funzione $\mathrm{F}$ e, quindi, ad una assimilazione interna della principessa con il donatore; del resto, anche Propp menziona la possibilità che il dono dell'oggetto magico si verifichi prima del danneggiamento che dà il via alla vicenda. Tuttavia, l'oggetto magico non serve affatto a far superare all'eroe le prove richieste per la conquista del bene desiderato, sarà al contrario l'occasione dell'infrazione di Gherardino e della sua ricaduta nella disgrazia. Considerando questo movimento in relazione a quello che seguirà, sembra invece di trovarsi davanti a una tipica sequenza da partie préparatoire: situazione di benessere - interdizione - allontanamento - trasgressione - disgrazia. Quello che rispetto alla sequenza precedente appariva come un "ritorno," assume in questa prospettiva l'aspetto di un "allontanamento" del protagonista, e la quête che seguirà servirà a far tornare Gherardino nel luogo che è ormai veramente suo, dove risiede la sua felicità, cioè nel regno della Fata.

In realtà, per quanto riguarda i primi due movimenti, appaiono più appropriate le categorie e gli schemi che $\mathrm{A}$. Dundes ha ricavato dall'esame dei racconti degli Indiani d'America: racconti che per il loro carattere primitivo presentano strutture più semplici di quella 
della fiaba, e non si differenziano ancora dal mito (del resto il carattere mitico della "storia di Lanval" è dato proprio dalla presenza della sposa soprannaturale). Seguendo gli schemi di Dundes, la formalizzazione dei primi due movimenti sarebbe dunque la seguente:

Primo mov.:

$\mathrm{L}-\mathrm{T}-\mathrm{T}-\mathrm{TA}-\mathrm{LL}$

Mancanza - Compito difficile - Superamento - Riparaz. della - mancanza

Secondo mov.:

L - LL - Int. - Viol. - Conseq.

Mancanza - Riparaz. della mancanza - Divieto - Infrazione - Conseguenze - dell'infrazione

Questa struttura mitica è stata integrata con alcuni elementi della struttura fiabesca: quelli cioè che nello schema del primo movimento abbiamo indicato $\operatorname{con} \beta^{2} \gamma^{1} \delta^{1} a(=L) t$.

Terzo movimento

1 Nuova partenza da Roma

Y Naufragio al passaggio di un fiume

D Apparizione di una fata (sorella della Fata Bianca)

Y Salvataggio in una rocca

Gherardino scompare su una barca incantata

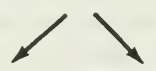

Viaggio in nave di Marco

Bello e della fata

Avventura d'amore su

un'isoletta

Arrivo dalla Fata Bianca

e informazione che

Gherardino è scomparso

A Gherardino imprigionato in Alessandria

$\S$ Una serva parla alla sultana del bel prigioniero

DE Liberazione dalla prigionia in virtù della sua abilità nel "servire"

Y Relazione Gherardino-sultana

$\S$ Bando del torneo 
F Dono di armi e sopravvesti

G Viaggio

M Torneo, di cui il Sultano è temporaneamente campione

O Gherardino arriva in incognito

U Uccide il sultano ("Punizione" rispetto al danneggiamento di questa sequenza)

N Gherardino risulta vincitore del torneo

Q Riconoscimento

$\mathrm{W}^{2}$ Gherardino sposa la Fata e resta signore del suo regno.

La mini-sequenza naufragio-apparizione della sorella-salvataggio in una rocca, e la conseguente vicenda di Marco Bello appaiono incongruenti rispetto all'intreccio lineare di una fiaba, e si potrebbero indicare con $\mathrm{Y}$ (elementi di origine "estranea"). Tuttavia questo episodio è essenziale all'intreccio perché offre a Gherardino l'occasione di imbarcarsi e di essere portato via da una tempesta motivo che prepara un'altra sequenza del racconto, per cui è sembrato di poter considerare la fata sorella come un Donatore. L'episodio trova probabilmente la sua necessità nel fatto che fin dall'inizio l'autore ha fatto accompagnare l'eroe da un amico, e deve dare un certo spessore anche alla storia di questo personaggio, costruendo dunque un racconto (fino a questo momento) a due fili, che si ricongiungeranno poi nell'ottava finale del cantare con le nozze comuni.

La sparizione di Gherardino sulla barchetta trasportata dalla tempesta serve invece funzionalmente a dare il via a una sequenza che appare coerentemente fiabesca fino alla fine del cantare. $\dot{E}$ interessante anzi osservare che, poiché la disavventura di Gherardino e la sua separazione dalla Fata Bianca cadono sotto la sua esclusiva responsabilità, manca cioè la figura - essenziale nelle fiabe - dell'aggressore, l'autore del cantare introduce un terzo, tenue, filo narrativo (la sultana innamorata di Gherardino) che fornisce al racconto la figura di un aggressore. Questi riceverà alla fine la dovuta punizione, e la sultana sarà il soggetto del terzo matrimonio dell'ottava finale.

Pur con questa struttura fiabesca, l'ultima parte del cantare (da A a $W_{0}^{2}$ ) è tutta tessuta con motivi cavallereschi: l'imprigionamento del guerriero cristiano, la serva che riferisce alla sultana della bellezza del prigioniero, la trasformazione di Gherardino in 
'servitore di coltello," la seduzione della moglie del sultano, il bando del torneo, ecc. L'unica sorpresa in questo tessuto coerentemente tradizionale-cavalleresco, è l'esito della seduzione della sultana: mentre il luogo comune vuole che il cavaliere respinga fermamente le avances della moglie del suo signore, in nome del suo vero amore, e a costo di essere esposto a una punizione ingiusta (cfr. Lanval, Tristano, Dama del Vergiù ecc.), Gherardino dopo un primo rifiuto si adatta di buon grado ad accontentare le voglie della signora; è questo un segno, si direbbe, dei tempi mutati (siamo ormai in pieno Trecento), e le novelle "boccaccesche" devono aver allentato il rigido codice delle virtù feudali. Ma è anche un segno della libertà con la quale l'autore del Bel Gherardino combinava ed elaborava liberamente i motivi fornitigli dalle sue fonti.

\section{Liombruno}

Ripete lo schema a tre movimenti del Bel Gherardino: nel primo movimento una serie d'avventure porta Liombruno, in un paese lontano, all'ottenimento dell'amore di una fata; il secondo movimento vede il ritorno a casa di Liombruno, con la sua trasgressione e la perdita della felicità; il secondo cantare - e terzo movimento - narra la quête attraverso la quale Liombruno torna al suo stato di felicità nel paese lontano.

Primo movimento

$\alpha \quad$ povertà di un pescatore

$\eta^{1} \quad$ patto col diavolo

1 consegna del figlio

Y Liombruno si fa il segno della croce: il diavolo fugge.

D Intervento di un'aquila che promette a Liombruno di riportarlo da suo padre e invece lo rapisce

$G^{1} \quad$ Volo

Y Educazione di Liombruno

$\mathrm{W}^{0}$ Matrimonio e felicità di Liombruno

Anche qui il nucleo fondamentale della "storia di Lanval" è fatto precedere da un preambolo di tipo fiabesco (il patto ingannatore di Propp). Questa partie préparatoire si riallaccia del resto in maniera piuttosto meccanica all'avvio della storia vera e propria: il fanciullo 
si fa il segno della croce e del primitivo "aggressore" non si sente più parlare. Il rapimento del fanciullo sul dorso di un'aquila, che si trasforma poi in principessa, è una novità assoluta rispetto alla tradizione della "storia di Lanval": l'autore ha probabilmente combinato qui motivi di provenienza eterogenea. Strutturalmente si ha l'assimilazione del "donatore," dell'"aiutante magico" e della "principessa" in un solo personaggio. L'educazione di Liombruno si rifà al motivo cavalleresco delle Enfances.

Secondo movimento

$\S \quad$ Nostalgia di Liombruno per la sua famiglia

$\mathrm{L}$

$\left(\mathrm{F}^{1}\right)$ Anello magico

LL

$\gamma^{\prime} \quad$ Proibizione di parlare della Fata

Int.

() Ritorno a casa

Y Torneo e vittoria di Liombruno

$\delta^{1} \quad$ Vanto

Viol

Y Liombruno condannato a morte

Conseq.

Y Apparizione provvidenziale di Aquilina

$\mathrm{AE}$

\section{A Aquilina abbandona Liombruno}

Anche qui, ricorrendo agli schemi elaborati dal Dundes per i miti primitivi, si ha una corrispondenza più precisa che utilizzando le categorie di Propp ( $\mathrm{L}=$ mancanza, $\mathrm{LL}=$ riparazione della mancanza: concorrono a formare questa categoria sia il ritorno a casa, che risponde alla nostalgia di Liombruno, sia le ricchezze procurate dall'anello magico, che sono il complemento indispensabile del ritorno; Int. = divieto, Viol. = infrazione, Conseq. $=$ conseguenze dell'infrazione, $\mathrm{AE}=$ conseguenze evitate).${ }^{18}$ Certi elementi della struttura mitica coincidono però con quelli della partie préparatoire di Propp (divieto, infrazione, danno), per cui questo secondo movimento ha anche la funzione di preparare il "danno" che darà il via alla quête fiabesca della seconda parte.

L'adattamento alla tradizione dominante del romanzo cavalleresco è evidente soprattutto nelle circostanze dell'infrazione, che ci presentano un torneo e una gara di vanti, oltre che nell'episodio relativo all'apparizione della fata per salvare Liombruno dalla condanna a morte, episodio che è già presente nel Lanval e che, 
secondo l'Hoeppfner, trova probabilmente la sua origine in un episodio del Tristan. ${ }^{19}$

Terzo movimento

$D^{\S^{\mathrm{vi}}} \quad$ Spartizione fra i tre malandrini

$\mathrm{F}^{\S} \quad$ Liombruno si impossessa degli oggetti magici

$\mathrm{D}^{2} \mathrm{E}^{7} \quad$ Incontro e colloquio con i mercanti

MN Raggiungimento della montagna inaccessibile

DEF Incontro con l'eremita e con i venti

G Viaggio insieme al vento

U Liombruno invisibile dà ad Aquilina segni della sua presenza

$\mathrm{QW}^{2} \quad$ Riconciliazione con Aquilina

Il terzo movimento segue in maniera ortodossa lo schema della fiaba. La lunga ricerca di Liombruno lo porta successivamente a contatto con tre "donatori," ciascun episodio conservando l'istituto della "prova" : nell'incontro con i malandrini Liombruno dà prova della sua furbizia, in quello con i mercanti della sua gentilezza, in quello con l'eremita dell'ortodossia della sua fede. Anche la figura dell'eremita, come già il diavolo della prima parte, sarà da considerarsi come una "substitution confessionnelle," ed è un luogo comune della letteratura cavalleresca. Nei "tiri" giocati da Liombruno invisibile ad Aquilina sarà probabilmente da riconoscere un elemento di "punizione," visto che Aquilina stessa aveva provocato il danno che sta all'inizio dell'ultima sequenza.

Ponzela Gaia

Nonostante lo schema della storia sia esattamente lo stesso dei due cantari precedentemente esaminati, la prima parte della Ponzela Gaia presenta una struttura compatta, più vicina alla "storia di Lanval," che non giustifica la suddivisione in due movimenti (manca in particolare l'elemento della lontananza meravigliosa del paese della fata); per cui il cantare si può dividere semplicemente in due movimenti. Osserviamo che l'episodio iniziale che serve ad agganciare il racconto al mito che conosciamo è di carattere non fiabesco ma romanzesco - per quanto degradato nell'assurdità di mettere a repentaglio la testa nella gara su chi riporterà la più bella 
cacciagione. Useremo anche questa volta come più pertinenti le categorie suggerite da Dundes.

Primo movimento

Corte di re Artù

Y Proposta di gara; Cacciagione di Troiano

Caccia di Galvano

$\mathrm{L}$

Combattimento con la biscia

T-TA

Amore della Ponzela Gaia e vittoria nella gara con Troiano LL

$\gamma^{1}$ La Ponzela Gaia proibisce a Galvano di parlare di lei Int.

Y Circostanze della trasgressione : fallito tentativo di seduzione della regina; torneo di vanti

$\delta^{1} \quad$ Infrazione del divieto

Viol.

Perdita dei favori della P.G. e condanna a morte di Galvano

Conseq.

La P.G. evita a Galvano la condanna a morte

$\mathrm{AE}$

A La Ponzela Gaia è imprigionata dalla madre

Con il danno subito dalla donna dell'eroe rientriamo ntllo schema della fiaba; e questa volta si ha anche un aggressore in piena regola nella madre della Ponzela Gaia. I segmenti indicati con $\mathrm{Y}$ appartengono esclusivamente alla sfera cavalleresca.

Pur nella configurazione cavalleresca e con le "substitutions littéraires" che sono particolarmente avvertibili in questo cantare, la seconda parte riprende lo schema della quête, con l'incontro di diversi aiutanti, il combattimento finale, la liberazione della principessa, la punizione dell'aggressore e il "ritorno a casa" :

Secondo movimento
1
Galvano parte alla ricerca della P.G.
Y
Incontro con Breus
DF
Incontro con le donzelle che piangono la sorte della Ponzela Gaia e indicano a G. il cammino

Dneg-pos. EF Incontro con una dama, dapprima ostile, poi amichevole 


$\begin{array}{ll}\text { HJ } & \text { Assedio ed espugnazione della città di Pela horso } \\ \text { DF } & \text { Stratagemma suggerito dalla P.G. per mezzo della serva } \\ \mathrm{K}^{10} & \text { Liberazione della Ponzela Gaia } \\ \text { U } & \text { Punizione della Fata Morgana } \\ \downarrow & \text { Ritorno alla corte di re Artù }\end{array}$

Come già per l'episodio iniziale (la gara di caccia fra Galvano e Troiano), il cantare della Ponzela Gaia utilizza largamente motivi tratti dai romanzi cortesi, tanto da nascondere quasi la matrice fiabesca della triplicazione degli incontri dell'eroe. Infatti, l'incontro con Breus, oltre ad essere un motivo di chiara derivazione tristaniana, non porta minimamente avanti la ricerca di Galvano, e resta un elemento isolato; l'incontro con le donzelle è ugualmente un motivo derivato da un episodio dell'Yvain di Chrétien de Troyes (le donzelle piangenti al castello della Pessima Avventura), ma serve almeno a mettere Galvano nella giusta direzione per la città dove ritroverà La Ponzela Gaia. Sembra mancare del tutto l'elemento della "prova," mentre nell'incontro con la dama lo scontro vittorioso di Galvano con i cento cavalieri appare il superamento della prova atto a trasformare il "donatore" da ostile in amichevole; l'aiuto sarà sempre, però, una semplice indicazione della strada, mentre il superamento della lotta finale rimane affidato esclusivamente al valore dell'eroe. Né è (tematicamente) più fiabesco lo stratagemma suggerito dalla Ponzela Gaia (che assume per l'occasione la funzione di "donatore") di travestire i suoi uomini da donne e di vestire i colori della Dama del Lago. L'episodio offre il destro all'autore del cantare di sfoggiare la sua erudizione a proposito dei rapporti di parentela fra Morgana, la Dama del Lago e Uterpandragone, e trova forse qui la ragione della sua invenzione; in ogni modo, lo stratagemma suggerito dalla Ponzela Gaia è veramente quello decisivo per portare a termine vittoriosamente la lotta, come lo sarebbe stato l'oggetto magico della fiaba.

La penetrazione di temi "letterari" nel tessuto della P.G. è dunque tale da intaccare notevolmente la superficie del racconto, senza giungere per questo a mascherare completamente la struttura soggiacente che resta riconoscibilmente mitica nella prima parte e fiabesca nella seconda.

\section{Gismirante}

In questo cantare le vicende che conducono all'acquisto e alla perdita della principessa sono precedute da un episodio preparato- 
rio, a sua volta preceduto da una vicenda introduttiva. Si hanno quindi i seguenti blocchi :

$\left.\begin{array}{l}\text { Vicenda introduttiva } \\ \text { Episodio preparatorio }\end{array}\right\}$

Primo movimento

Acquisto della principessa $\}$

Secondo movimento $\left.\begin{array}{l}\text { Perdita della principessa e vicende } \\ \text { che conducono alla sua riconquista }\end{array}\right\}$ Terzo movimento

\section{Primo movimento}

$\alpha \quad$ Cortese ha un figlio

$\beta^{2} \quad$ Cortese muore

$\boldsymbol{\gamma}^{2}$ raccomandando al figlio di tornare alla corte di Artù

$\delta^{2} \quad$ Gismirante è accolto in corte

$\S \quad$ Usanza della corte

a per cui tutti restano senza mangiare

1 Gismirante si mette alla ricerca di "fresche novelle"

D Incontra una fata

K che gli dà un capello d'oro e gli racconta dello strano costume della principessa

1 Gismirante torna a corte

Come si vede, la struttura del primo movimento è coerentemente fiabesca. Manca, come nella maggior parte dei nostri cantari, la funzione della "prova" esplicitamente imposta dal "donatore"; la funzione $\mathrm{F}$ in questo caso si identifica con $\mathrm{K}$; si tratta anche in questo caso di un elemento verbale, cioè di una notizia, come lo erano le indicazioni sulla strada da seguire del Liombruno e della Ponzela Gaia.

Secondo movimento 
$\delta^{7} E^{7} \quad$ aiuta un grifone nella lotta contro un drago

$\mathrm{L}^{7} \mathrm{E}^{7}$ dà da mangiare a un'aquila affamata

$\delta^{7} E^{7} \quad$ libera uno sparviero

$D^{2} E$ incontra una fata che tenta di dissuaderlo dalla sua impresa. G. resta fermo nel suo proposito

$F^{1} \quad$ La fata gli dà un cavallo velocissimo

G G. giunge a destinazione

Y Arrivato nella città designata, G. si fa notare per la sua bravura nel giostrare ma non riesce a vedere la principessa

M La vigilia di San Martino si reca in chiesa e parla alla principessa

N La dama si intenerisce e dà appuntamento $\mathrm{a}$ G. per la notte

(F) La fanciulla prende con sé una bacchetta magica

Fuga dei due

$\mathrm{P}_{\mathrm{r}}^{6} \quad$ Inseguimento del padre e dei suoi uomini

$R_{\mathrm{s}}{ }^{2} \quad$ La fanciulla passa il fiume con la bacchetta magica.

Gismirante resta a combattere gli inseguitori

U Vittoria sul padre della fanciulla

Nell'incontro di Gismirante con la fata, par bene di riconoscere una "prova" nel tentativo operato dalla fata e stornato da Gismirante, di dissuaderlo dal perseguire il suo intento; tanto è vero che al rifiuto di G. segue il dono del cavallo, e anche nel terzo movimento ad ogni replica del rifiuto segue un aiuto, più o meno concreto, da parte della fata. Forse è questo il senso segreto del motivo, che troviamo anche nella Ponzela Gaia e nel Gibello, della dama che offre all'eroe il suo amore e, respinta, anziché offendersene, aiuta l'eroe a perseguire il suo intento.

La combinazione di motivi tolti da fonti diverse è evidente in questo movimento nella funzione del cavallo che, dopo aver portato G. in tre giorni dove un cavallo normale non sarebbe arrivato in dieci anni, sembra perdere tutta la sua virtù motoria al momento della fuga dei due innamorati, che si fanno ben presto raggiungere dagli inseguitori. La bacchetta magica sembra ugualmente un'interpolazione, visto che non assolve alcuna funzione veramente necessaria, e gli inseguitori sono sgominati dal valore di Gismirante, sia pure con l'aiuto del cavallo. É ormai evidente che, 
nei nostri cantari, le "imprese difficili" (la "lotta col dragone" di Propp) sono sostituite da imprese tipicamente epico-cavalleresche : tornei, combattimenti, assedi.

\section{Terzo movimento}

( $\beta$ G) Gismirante si addormenta

A L'Uomo Selvaggio rapisce la principessa

$\mathrm{R}_{\mathrm{s}}{ }^{1}\left(\mathrm{~F}^{9}\right)$ Il grifone trasporta Gismirante al di là di un corso d'acqua

DEF G. ritrova la fata che lo consiglia su come riottenere la fanciulla

$\S \quad$ G. chiede alla fanciulla di informarsi su dove l'Uomo Selvaggio tiene il cuore. Dopo una prima informazione menzognera, l'Uomo Selvaggio glielo rivela e la fanciulla lo comunica a Gismirante

DE Nuova prova di fermezza : la fata invita G. ad abbandonare la fanciulla al suo destino; al suo rifiuto, lo incoraggia ad andare a Roma, implicitamente promettendogli il suo aiuto.

1 Gismirante parte per Roma

O Diventa scudiero di un cavaliere

$\mathrm{B}^{4} \quad$ Il re deve offrire il proprio figlio in pasto al "porco troncascino"

C Gismirante si vanta di poter uccidere il porco

F Riceve dal re buone armi e buon cavallo

$\mathrm{HJ}\left(\mathrm{F}^{9}\right)$ Lotta con il porco, la lepre, il passerotto, superata con l'aiuto dell'aquila e dello sparviero

Y Gismirante rifiuta la mano della principessa

$\downarrow$ Gismirante torna dalla fata

F La fata gli suggerisce il modo di entrare nel castello senza porte

(DF) L'Uomo Selvaggio dà alla fanciulla un anello che permette di vedere la porta

U Appena entrato, G. uccide il passerotto

$\mathrm{K}^{10} \quad$ Liberazione della fanciulla e di tutte le donne prigioniere dell'Uomo Selvaggio

$\downarrow$ Ritorno a corte

$W^{0} \quad$ Nozze 
Durante il ritorno, l'eroe subisce un nuovo danneggiamento, che dà il via a una seconda sequenza della fiaba. Gli aiutanti della prima parte del racconto (grifone, aquila, sparviero) ricompaiono in questa seconda parte a pagare il loro debito di riconoscenza, il che sottolinea l'unità del lungo racconto. Alla storia della liberazione della fanciulla s'intreccia l'altra vicenda della liberazione della città di Roma (non si caposce perché Gismirante, arrivato a Roma, non sia andato subito in cerca del porco troncascino e abbia invece aspettato che la città fosse in lutto, passando il tempo a servire come scudiero); il che costringe Gismirante a rifiutare la mano della figlia dell'Imperatore per poter tornare a concludere l'altra storia. L'aggressore adempie, temporaneamente, alla funzione di donatore quando dà alla fanciulla l'anello che permette l'ingresso nel castello; l'eroe e l'eroina collaborano, in questa sequenza, alla vittoria sull'aggressore. ${ }^{20}$

\section{Gibello}

Il Gibello è il più "cavalleresco" e il meno "meraviglioso" fra i cantari del nostro corpus. Niente animali parlanti, niente oggetti magici. Tuttavia la vicinanza dei suoi motivi a quelli degli altri cantari considerati e la possibilità di riconoscere nel racconto sequenze fiabesche permettono di includerlo nella categoria del "cantare fiabesco" italiano.

Formalmente è costituito da un solo canto, ma anche qui è possibile riconoscere tre movimenti. Nel primo il bambino portato ad affogare non solo viene salvato ma raggiunge, con l'età, benessere, prestigio e l'amore della principessa. L'intervento di un cavaliere invidioso, rivelando l'oscurità delle origini di Gibello, determina la sua partenza alla ricerca della famiglia. L'autore segue per un certo tratto il viaggio di Gibello che, dopo aver abbattuto due cavalieri ed essersene acquistata la fedeltà, finisce in una prigione per la gelosia preveggente di un duca, vassallo del padre di Gibello. A tanto, l'autore abbandona il nostro eroe nella sua prigione e torna a riprendere il filo dei genitori di Gibello (secondo movimento), che vogliono dar moglie all'altro loro figlio e scelgono come sposa, guarda caso, proprio la principessa promessa sposa di Gibello. Al suo rifiuto, il pretendente respinto assedia la città della principessa e Gibello, che lo viene a sapere, approfitta dell'accondiscendenza della duchessa per uscire temporaneamente di prigione, correre a liberare la principessa con l'aiuto dei due cavalieri prima sottomessi, e uccidere il marito della duchessa. Dopo di che, ritorna lealmente in prigione. ${ }^{21}$ Così, dunque, la 
principessa è liberata ma è lontana, Gibello si trova nel regno di suo padre ma senza saperlo, e in prigione. L'innamoramento della duchessa, benché rifiutato da Gibello, innesta il terzo movimento e imprime una svolta decisiva alla vicenda : la duchessa conduce con sé Gibello a una grande festa indetta dal padre di Gibello e, una volta a corte, la rassomiglianza dell'eroe col figlio del re porta al riconoscimento di Gibello. Ma la storia non è ancora conclusa perché deve portare i suoi personaggi a superare quell'ostacolo che aveva originato la vicenda, e cioè l'erronea credenza del padre dell'eroe che il parto gemellare denunciasse un adulterio. Sciolto con un banale sermoncino anche quest'ultimo ostacolo, Gibello fa venire a corte la principessa e ricongiunge i due scopi della sua esistenza (in quanto personaggio fiabesco), ottenendo il matrimonio con la sua bella e ereditando il regno di suo padre. In effetti, le nozze e "la metà del regno" sono spessissimo il punto d'arrivo delle fiabe, ma il Gibello ha fatto dei due scopi supremi e congiunti della fiaba due scopi separati, ciascuno dei quali è ottenuto con una sequenza fiabesca. Conviene quindi formalizzare, piuttosto che $\mathrm{i}$ tre movimenti della costruzione del cantare, le due sequenze fiabesche che in ciascuno dei movimenti vengono intrecciate.

Quête del regno

$\begin{array}{ll}\text { Y } & \begin{array}{l}\text { Erronea credenza che il parto gemellare denunci un } \\ \text { adulterio }\end{array} \\ \text { A }^{13} & \text { Uno dei gemelli è portato ad annegare } \\ \text { B }^{5} t & \text { Il bambino è portato via dai mercanti } \\ \text { DF } & \begin{array}{l}\text { La principessa Argogliosa lo accoglie e gli fa dare } \\ \text { un'educazione }\end{array} \\ \text { Dneg } \rightarrow \text { pos. } & \begin{array}{l}\text { Gibello finisce imprigionato dal duca di Serpentina ma } \\ \text { la duchessa sinnamora di lui }\end{array} \\ \text { F } & \text { e lo conduce alla corte dei genitori } \\ \text { ICK } & \text { Qui Gibello riconosce sua madre } \\ \text { H } & \text { ma deve lottare contro il padre e la sua iniqua sentenza } \\ \text { J-U } & \text { Vittoria di Gibello e cambiamento d'idea del padre } \\ 2^{\circ} \mathrm{K} & \text { Gibello riconosciuto come figlio del re e erede del regno }\end{array}$

Quête della principessa 
DE Cavaliere Nero

DE Cavaliere Vermiglio

Dneg $\rightarrow$ pos. Duchessa di Serpentina

A

Ft

$\mathrm{F}^{9}$

$\mathrm{K}^{10}$

$\mathrm{W}^{0}$ contr.
Assedio della principessa da parte del pretendente respinto

Gibello, liberato, parte

Raccoglie lungo il viaggio le truppe del Cavaliere Nero e del Cavaliere Vermiglio

Combattimento e vittoria contro gli assedianti

Liberazione della principessa

Come già per gli altri cantari, abbiamo usato la sigla $\mathrm{F}$ per indicare un aiuto offerto al protagonista dal "donatore"; aiuto che porta avanti il filo del racconto, ma che è la funzione fiabesca più propensa ad assumere una configurazione razionalizzata e cavalleresca.

Raccogliendo le fila di tutte le osservazioni sparse nel corso dell'esame analitico, riconosceremo dunque che nel cantare italiano è ancora possibile rinvenire episodi di struttura "mitica," che sono quelli risalenti alle fonti francesi di alcuni secoli prima ( $\mathrm{L}-$ LL - Int. - Viol. - Conseq. - AE). Tuttavia, nel momento in cui fra le "conseguenze dell'infrazione" e il riottenimento del bene perduto si inserisce la quête, siamo passati da una struttura mitica ad una struttura fiabesca, in cui l'episodio mitico diventa l'antefatto della fiaba. L'assimilazione del cantare alla fiaba si fonda sui seguenti elementi caratteristici : 1) c'è sempre un antefatto che dà luogo ad una quête; 2 ) il racconto tende al raggiungimento dell'oggetto della quête descrivendo incontri dell'eroe con personaggi che gli danno i mezzi di perseguire il suo fine; 3 ) il racconto è completo quando il bene, perduto nel corso della favola, è stato riconquistato dall'eroe.

Tuttavia la favola cavalleresca del cantare italiano presenta dei tratti peculiari rispetto allo schema della "fiaba classica di magia," che gli derivano da un lato dalla sua vicinanza al romanzo cavalleresco, dall'altro dalla sua derivazione dal lai francese. Per esempio, la fiaba classica di magia prevede per l'eroe la partenza da 
un ambiente domestico e, dopo il raggiungimento dell'oggetto della quête, il suo ritorno all'ambiente di partenza. I cantari che hanno conservato più fedelmente il ricordo del lai francese, hanno invece un movimento pendolare che si conclude nel regno "altro," o lontano, anziché nel "regno" di partenza. Così nel Bel Gherardino e nel Liombruno:

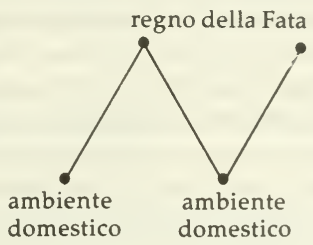

Interessante osservare che nella Ponzela Gaia, nonostante la fedeltà del rimaneggiatore alle fonti antiche, la struttura fiabesca ha preso il sopravvento e il "ritorno" dell'eroe è un ritorno al punto di partenza (la corte di re Artù). Stessa cosa nel Gismirante e nel Gibello, dove d'altra parte la conservazione dei motivi mitici è molto più mediata.

Nei nostri cantari troviamo immancabilmente i personaggi dell'eroe, della principessa e degli aiutanti (quasi sempre più di uno). Gli "incontri" dell'eroe nel corso della sua quête sono, normalmente, tre : così nel Liombruno (malandrini - mercanti - venti), nella Ponzela Gaia (Breus - damigelle piangenti - dama fedele alla Ponzela Gaia), nel Gibello (cavaliere Nero, Cavaliere Vermiglio, duchessa di Serpentina). Nel Gismirante l'incontro con un "donato$\mathrm{re}^{\prime \prime}$ fondamentale (la fata), è combinato con i tre incontri successivi con il grifone, l'aquila e lo sparviero. Il personaggio dell'aggressore ha invece nei cantari una consistenza piuttosto fluida : il punto di partenza delle nostre storie è spesso, infatti, una mancanza piuttosto che un danneggiamento da parte di un aggressore costituito, per cui vengono introdotti nel corso del racconto degli aggressori episodici, contro i quali far rifulgere il valore dell'eroe : il Sultano del Bel Gherardino (il duca del Gibello), il padre della principessa e il "porco troncascino" nel Gismirante; fanno eccezione la fata Morgana della Ponzela Gaia e il padre di Gibello nel cantare omonimo, che assolvono alla vera e propria funzione fiabesca dell'aggressore. Mancano completamente, nei nostri cantari, i personaggi del mandatario e del falso eroe. L'elemento dell'auxiliaire magique è fluido e notevolmente ridimensionato: l'aiuto ricevuto dall'eroe nel perseguimento del suo fine è spesso di carattere verbale (un'informazione), o consiste negli strumenti necessari alla "lotta epica" (Bel Gherardino, Gismirante), mentre il superamento della "prova finale," 
che prelude alla conquista o alla riconquista del bene desiderato, è affidato in genere al valore personale dell'eroe (Bel Gherardino, Ponzela Gala, Gibello). L'unica eccezione è il Liombruno, in cui gli oggetti magici (gli stivali "delle sette leghe," il mantello che rende invisibili) sembrano veramente essenziali al perseguimento del fine di ritrovare la principessa e riconquistare il suo amore (e la dimostrazione del valore epico di Liombruno è affidata all'episodio del torneo); nel Gismirante gli uccelli fatati hanno una funzione decisiva soltanto nel caso della lepre e del passerotto, mentre gli altri oggetti magici, pur presenti a profusione, hanno una funzione episodica e, tutto sommato, secondaria: come il cavallo velocissimo, la bacchetta magica, l'anello dell'Uomo Selvaggio.

La configurazione cavalleresca della prova finale trova una bizzarra conferma in un cantare che non solo non è compreso nel nostro corpus ma non può neanche essere considerato un cantare fiabesco, essendo in sostanza il rifacimento in ottave di una novella del Boccaccio : la novella 9" del 2" giorno racconta di come la sposa di Bernabò da Genova, ingiustamente accusata di adulterio da un impostore, e sfuggita per miracolo alla morte che il marito le aveva decretato, si travesta da uomo, diventi la persona di fiducia del Sultano di Alessandria e, in questa veste, riesca a far confessare all'impostore le sue bugie e a tornare serenamente a Genova col marito. Nel cantare pubblicato dal Levi col titolo di Madonna Elena la prova dell'innocenza della donna è offerta da un "giudizio di Dio" che consiste in un alquanto improbabile duello fra la donna stessa, in abiti maschili, e l'impostore - duello vinto naturalmente senza sforzo dalla donna: evidentemente, le "leggi del genere" hanno operato in questo caso anche di fronte ad una fonte che portava una conclusione totalmente diversa.

\section{ANALISI STRUTTURALE : IL ROMANZO CAVALLERESCO}

In ogni modo, la struttura mitico-fiabesca, sia pure con adattamenti alla tematica cavalleresca, non esaurisce l'esame dei nostri cantari. Come abbiamo già osservato, un elemento costante che non ha riscontro nella fiaba, è l'attenzione accentuata al valore personale dell'eroe, che si rivela non soltanto nel privare il protagonista di ogni aiuto soprannaturale nel corso delle sue prove, ma anche nell'insistere sulla sua formazione, sulle sue prove d'iniziazione, sulla sua capacità di attrarre l'amore delle più alte dame. In altri termini, il racconto del cantare cavalleresco non tende più soltanto alla realizzazione di una sequenza rigida di funzioni, com'è il caso della fiaba, ma anche alla messa in evidenza di determinati valori accentrati nel personaggio principale : è il procedimento che si 
dispiega nei poemi delle Enfances degli eroi epici, qui condensato e rappreso nei brevi limiti che il cantare concedeva all'elaborazione delle sue storie. Sembra che debbano assolvere a questa funzione lo scontro di Gherardino e Marco col serpente e con l'orso, e lo scontro di Galvano con la "serpa" fatata. Dal canto loro, Liombruno e Gibello traggono i migliori frutti dall'educazione che rispettivamente Aquilina e Argogliosa fanno dare ai loro protetti; e anche Gismirante
... .serviva sì ben, che l'avìe caro
il re Artue sopr'ogni damigello
e tutti i cavalieri innamorâro
tanto egli era apariscente e bello;
ed insegnârgli giostrare e schermire
sì che fu sopra ogn'altro pien d'ardire. (Gism., 1, 5, 3-8)

Più tardi, Gherardino si attirerà l'amore di una sultana e il benvolere del sultano per la sua compiutezza del "servire di coppa e di coltello." ${ }^{23}$ Ugualmente Gibello, Gismirante e Galvano si attirano l'amore di una dama, o fata che sia, diversa dalla principessa; e Liombruno sfiora il matrimonio con un'altra principessa.

Tuttavia, il mondo del romanzo cavalleresco offre al cantare qualcosa di più profondo che un repertorio di motivi e di valori. Sembra in effetti di poter riconoscere in questo o quel cantare degli elementi di struttura che lo avvicinano alle modalità di svolgimento proprie del romanzo cavalleresco. ${ }^{24}$

Come nel romanzo cavalleresco, certe "funzioni," o tappe dell'azione, che nella fiaba vengono svolte rapidamente, assumono uno svolgimento ricco di articolazioni tanto da costituire delle vere e proprie sequenze. In quattro dei nostri cantari l'intero primo canto è in effetti dedicato alla preparazione della disgrazia che apre il movimento della quête; ma esso è articolato in modo più ampio della partie préparatoire di Propp e si avvale di sequenze proprie del "corpo" della fiaba o del mito. Allo stesso modo, nel $1^{\circ}$ libro dei Reali, il primo movimento costituisce, tutto intero, l'antefatto del secondo movimento, e i due movimenti hanno poi una conclusione comune; nel $3^{\prime \prime}$ libro, i fatti narrati nel primo e nel secondo movimento possono rapppresentare, tutti insieme, la funzione di "allontanamento" dell'eroe rispetto al terzo movimento dello stesso libro.

Come nel romanzo cavalleresco, nei cantari il raggiungimento dell'oggetto del desiderio può arrivare a tappe. Per esempio, Fiovo scacciato dal padre per aver ucciso Saleone (infrazione) passa attraverso la tipica trafila fiabesca D E F H J per giungere alle nozze e al trono, che sono normalmente la conclusione della fiaba; ma poiché il danno iniziale era costituito in questo caso dall'inimicizia 
col padre, è necessaria un'altra sequenza (Costantino, padre di Fiovo, assediato dai pagani, manda a chiamare il figlio, che accorre in suo aiuto e, l'incognito Riccieri agendo come aiutante, vince $\mathrm{i}$ nemici e libera Roma) per giungere, prima, alla riconciliazione col padre, e poi allo scopo specifico di questa sequenza e cioè la liberazione di Roma. Allo stesso modo, Gibello ottiene prima l'amore della principessa, poi il riconoscimento della madre, poi la riconciliazione col padre, e finalmente le nozze.

Altro tratto del romanzo cavalleresco che trova nei cantari in cui si manifesta una sua forma particolare, è la presenza nel racconto di più fila, che vengono tutte raccolte e portate a conclusione. Nel romanzo, però, le conclusioni relative alle varie fila possono essere sfalsate (prima si conclude una vicenda, poi se ne conclude un'altra); nel cantare, queste tendono a raccogliersi tutte in un'unica conclusione, che si trova, ovviamente, alla fine del cantare. Così il Bel Gherardino, che intreccia la vicenda principale di Gherardino e della Fata Bianca con quelle di Marco Bello e la sorella della Fata, e anche della Sultana innamorata di Gherardino:

Pò ch'a la fata ebbe dato l'anello,

grande festa fae, che.ll'hae ricognosciuto.

E la serocchia diede a Marco Bello,

e ha.lo sempre con seco tenuto;

e quella del Soldano diede a uno donzello

di grande legnaggio, cortese a saputo;

e novanta anni vivette signore.

Questo can to è compiuto a vostro onore. (Bel Gher, 11, 47)

Il Gibello segue fondamentalmente le vicende della vittima ma, in via subordinata, narra anche quelle del persecutore, oltre a dare una certa individualità alle vicende dell"'aiutante" (la duchessa). Anche qui le ultime due strofe richiamano e sistemano le vicende di tutti i personaggi principali:

Il buon Gibello con allegro cuore

isposò la pulzella innanzi al padre.

morta d'amore cade la duchessa.

chiamato fu signor di tutto, assiso,

poi che 'l padre passò di questa vita,

e'l fratel per signor d'altre contrade. (Gib., 86, 1-2,8; 87, 5-7)

Ma il procedimento che più degli altri ci sembra ricollegare i nostri cantari al romanzo cavalleresco quale è venuto costituendosi all'altezza cronologica dei Reali di Francia è il ritmo di "acquisti" $e$ "perdite" che si trova sporadicamente nel romanzo e che diventa invece il procedimento unificatore e probabilmente distintivo del cantare. Si osservi infatti come viene condotto avanti il racconto 
nel 30 libro dei Reali dedicato alle vicende di Riccieri e Fegra Albana : la principessa Fegra Albana, innamorata di Riccieri per fama, lo manda a chiamare tramite un messo, introducendo così nella vita di Riccieri una situazione di mancanza.

Riccieri giunge da Fegra Albana e vince la giostra, il cui palio sarebbe la mano di Fegra - sembra dunque aver quasi raggiunto l'oggetto del desiderio; ("acquisto")

senonché è scoperto come cristiano e gettato in prigione. ("perdita")

Fegra Albana riesce a liberarlo e a farlo fuggire. ("riparazione della perdita")

Fegra è gravemente minaceiata dal fratello e manda a chiedere aiuto a Riccieri; ("perdita")

Riccieri è riconosciuto da Fegra e vince i suoi nemici. ("acquisto")

Si allontana di nuovo da Fegra per andare a visitare il Soldano. Fegra, ricevuta la falsa notizia della morte di Riccieri, si uccide. ("perdita")

Nel $4^{\circ}$ libro dei Reali Fioravante, esiliato dal suo regno, sente grida di aiuto e accorre a liberare la principessa Uliana dalle mani dei saraceni. ("acquisto")

Immediatamente dopo, sta per soccombere nella lotta con Finaù, che vuole ritogliergli la principessa ("perdita")

ma viene soccorso da Riccieri, e si impadronisce della spada Durlindana. ("acquisto")

Un falso pellegrino propina un sonnifero a Riccieri e a Fioravante e rapisce Uliana ("perdita")

ma per opera di un oggetto magico i due eroi si svegliano e liberano la principessa. ("acquisto")

Il saraceno Mambriano e i suoi uomini impegnano gli eroi in combattimento e rapiscono di nuovo Uliana. ("perdita")

I "nostri" ricevono soccorso da Tibaldo di Lima; recuperano Uliana e si recano tutti alla corte del padre di Uliana. ("acquisto")

Qui Tibaldo rischia di diventare nemico di Fiovo per l'amore di Uliana (minaccia di "perdita")

ma Fioravante rinuncia alla mano della principessa e riconquista l'amicizia di Tibaldo. ("perdita" sventata) 
Se ora torniamo ai nostri cantari e osserviamo il modo di procedere dei loro racconti, assisteremo ugualmente a una catena ininterrotta di "alti" e di“bassi," rispetto alla "felicità" dell'eroe; con la differenza che nel cantare il finale deve trovarsi in "alto" $\mathrm{e}$ che deve avere un carattere conclusivo, più di quanto non l'abbiano le vicende finali delle sequenze del romanzo, che preludono continuamente a nuovi svolgimenti nei libri successivi.

\section{Bel Gherardino}

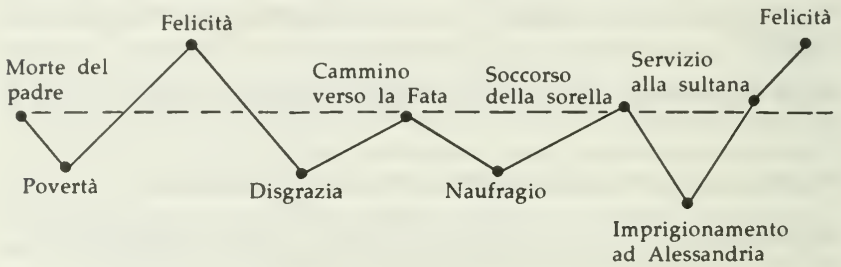

Liombruno

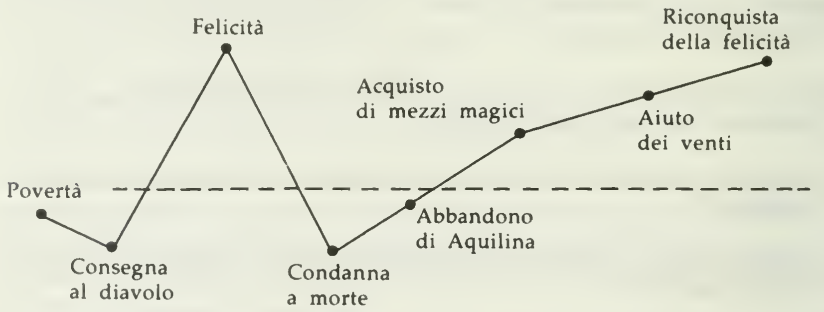

Ponzela Gaia

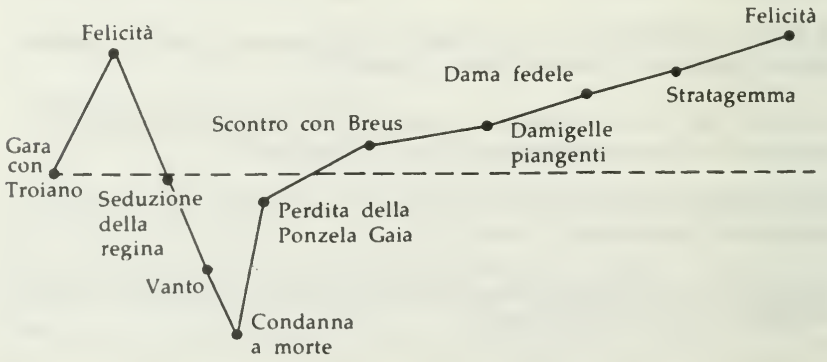


Gismirante

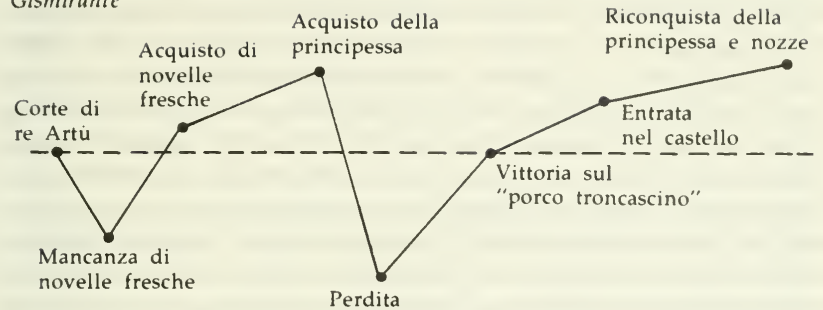

della principessa

Gibello

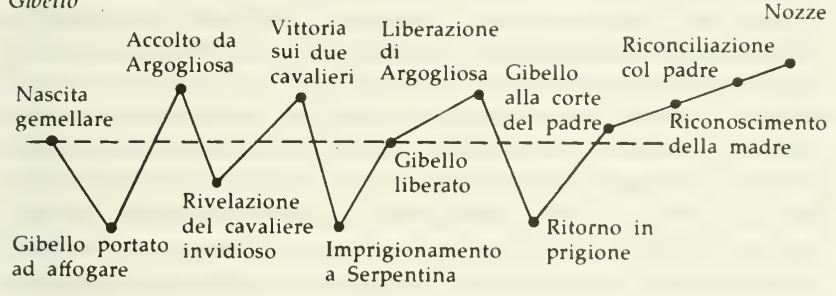

La difficoltà delle imprese nel Liombruno e nel Gismirante, gli ostacoli che si frappongono a Galvano lungo il suo cammino (nella fase della Quête) adempiono alla funzione di "suspence" che è ottenuta negli altri cantari dall'imprigionamento dell'eroe. Questo procedimento ritmico appare come un tratto specifico del cantare, capace di unificare i diversi tipi strutturali (mito, fiaba, romanzo) che convergono nella sua composizione.

Altro tratto specifico del cantare potrebbe rinvenirsi nel modo di iniziare la vicenda. Per la fiaba, Propp è riuscito a individuare delle modalità specifiche della partie préparatoire; queste non corrispondono sempre alle vicende iniziali dei nostri cantari. Ma neanche le vicende iniziali del poemi epici e dei poemi cavallereschi ci sono di maggior aiuto. E. Dorfman ${ }^{25}$ identifica in un inganno, o tradimento, la prima tappa di ogni poema epico da lui studiato. Del resto, i poemi e i romanzi cavallereschi si distendono per loro natura in movimenti cronologicamente e formalmente lunghi, mentre il cantare, come la fiaba, ha bisogno da un lato di far partire rapidamente l'azione; dall'altro, deve dare immediatamente l'impressione di un racconto pieno di fatti, in cui accadono molte cose. Forse è per questo che più di un cantare sembra avere due inizi, o 
meglio: alla vicenda che muove l'azione propria del cantare viene premessa un'altra introduzione, spesso di tipo fiabesco. E infatti: nel Bel Gherardino un padre muore lasciando a tre figli la sua eredità; e questo è un attacco tipicamente fiabesco che di solito viene associato o all'estrema miseria dell'eredità lasciata, o a un comando dato dal padre morente. Il Bel Gherardino gli fa succedere invece un'altra vicenda, che è quella che dà veramente il via all'azione: il carattere estremamente "cortese" di Gherardino lo riduce in miseria e i suoi fratelli non vogliono più aver a che fare con lui. Mosso dalla povertà Gherardino parte: la prima parte, e cioè la morte del padre, è inutile all'intreccio. Il Liombruno ha un inizio tipicamente fiabesco: il padre, poverissimo, promette a un essere maligno di consegnargli il figlio, in cambio del benessere. Questa prima vicenda viene però sbrigativamente conclusa col segno della croce che scaccia il maligno, e l'avvio della vicenda si ha veramente col rapimento del fanciullo da parte dell'aquila. Il Gismirante ha pure un inizio fiabesco: il padre dell'eroe muore e gli ordina di raggiungere la corte di Artù. Ma la vicenda ha inizio veramente soltanto quando Gismirante è già servitore a corte ed è provocata dallo strano costume in uso presso i cavalieri della Tavola Rotonda, di non poter mangiare se non arrivano notizie fresche. Il Gibello e la Ponzela Gaia hanno invece una vicenda iniziale che dà effettivamente inizio alle avventure dei protagonisti. Tuttavia, né le vicende iniziali del Gibello e della Ponzela Gaia né la vera vicenda iniziale degli altri tre cantari si iscrivono chiaramente negli schemi fissati da Propp. Rivediamoli schematicamente:

Bel Gherardino Per il suo carattere "cortese" Gherardino cade in disperata povertà e viene abbandonato dai fratelli.

Liombruno Un'aquila rapisce il fanciullo, lontano da casa, e lo porta nel suo regno.

Ponzela Gaia Galvano e Troiano impegnano la testa su chi di loro riporterà la più bella cacciagione.

Gismirante I cavalieri della Tavola Rotonda non possono mangiare per mancanza di notizie fresche.

Gibello Un re, convinto che il parto gemellare sia prova di adulterio, fa mettere a morte le donne che partoriscono gemelli. La regina partorisce due gemelli.

Salvo per l'inizio del Liombruno, tutte le altre vicende hanno una caratteristica in comune: quella di avere a che fare con convenzioni; 
con regole di comportamento imposte dagli uomini (dalla società, dall'autorità) a se stessi, come regole di un giuoco, e collocate al principio del racconto perché si intuisca subito che i fatti della vita, nella loro inesauribile complessità verranno inevitabilmente a trovarsi in contrasto con queste convenzioni, e forzeranno l'eroe ad abbandonare l'ambiente iniziale. ${ }^{26}$ Gherardino, che ha una visione "cortese" del mondo e dona con esagerata larghezza i suoi beni, è costretto dalla realtà delle circostanze a cadere in miseria a trovarsi così nella situazione di grave mancanza che provoca la partenza. Galvano e Troiano mettono in gioco la loro stessa testa (assai sconsideratamente, si direbbe!) per una scommessa su chi porterà la cacciagione più bella! Ma questo costringe immediatamente l'attenzione a concentrarsi sulle vicende di questo gioco che ha una posta tanto preziosa. La "mancanza" alla corte del re Artù, nel Gismirante, è anch'essa provocata da un elemento il più convenzionale possibile: il costume, imposto a se stessi dai cavalieri della Tavola Rotonda, di non mangiare se non si siano avute in giornata notizie interessanti! Ė evidente che la realtà non può corrispondere in ogni momento alle regole che gli uomini vorrebbero imporle, e che un giorno o l'altro le notizie mancheranno. Questo provoca la partenza di Gismirante e la sua ricerca d'avventure. Anche nel Gibello si trova un elemento di convenzione umana quale può essere una legge di $\mathrm{Re}$ - basata in questo caso su una credenza che si dà fin da principio per erronea: non appena informato, l'ascoltatore non può che aspettare il momento in cui il canterino racconterà che cosa è avvenuto che contravvenga a quella credenza, incorra nei rigori della legge, e dunque debba dar luogo a una partenza.

Questo non vuol dire che tutti i cantari del tipo cavallerescofiabesco cominciassero, o dovessero cominciare, con la "posa" di una regola convenzionale che i fatti avrebbero contraddetto per dare il via all'azione della storia, ma piuttosto che ci pare che un avvio di questo genere sia del tutto appropriato e funzionale a un racconto che, come la fiaba, deve fin dall'inizio creare una tensione da cui far scaturire, in modo naturale e prevedibile, il filo di un'azione; ma che svolge i suoi tempi non nell'ambito familiare e domestico che è quello della fiaba ma nell'ambito di una società civile - sia pur essa quella fittizia della letteratura cavalleresca, organizzata con i suoi ordinamenti, i suoi canoni e i suoi valori. L'esigenza riconosciuta da Propp di porre all'inizio di una storia una struttura atta a far verificare un danno o una mancanza resta qui la stessa; solo, le modalità per arrivare a questa perdita possono avere nel cantare una configurazione diversa dalla fiaba, e pare specifico del cantare il ricorso ad una convenzione sociale. 
Un'ultima caratteristica che additeremo come specifica del cantare è la compattezza della sua articolazione interna. Già abbiamo visto che se ci sono diversi fili il cantare li porta tutti a conclusione. Resta da dire che il cantare tende a un massimo di avventure con il minor numero di personaggi e di luoghi possibile: la vicenda di Gherardino si svolge tutta alternativamente nel suo luogo natale e nel regno della Fata, con l'intermezzo della prigionia in Alessandria, dove però lo raggiunge facilmente la notizia del torneo bandito dalla Fata. Allo stesso modo Liombruno, partito dalla sua casa e raggiunto il regno di Aquilina, torna nella sua casa e ritorna, alla fine del cantare, nel regno di Aquilina verso il quale lo ha portato senza deviazioni la sua quête; col breve intermezzo del torneo a Granata, regno che, come dicono i parenti di Liombruno, "sta qui appresso." La struttura della Ponzela Gaia è, da questo punto di vista, un po' più dispersiva, con una struttura più "lineare": vediamo Galvano, uscito dalla corte verso la foresta, avere la sua avventura con la Ponzela Gaia; tornato a corte, ivi ha luogo l'episodio della seduzione della regina e il vanto di Galvano, dopo di che, Galvano deve mettersi alla ricerca del regno della Fata Morgana, dove è imprigionata la sua bella. Le sue avventure si susseguono l'una all'altra senza rapporto fra di loro; solo, una volta liberata la Ponzela Gaia, Galvano si ferma, sulla via del ritorno a corte, al castello della dama fedele che l'aveva aiutato. Anche il Gismirante sembra a prima vista abbastanza dispersivo: venuto da Roma alla corte di re Artù, dopo la breve vicenda dell'avventura che permette a tutti i cavalieri di mettersi finalmente a tavola, anche Gismirante parte per il paese lontano della bella dai capelli d'oro; più tardi, sulla via del ritorno, sarà costretto a una nuova digressione per poter catturate il cuore dell'Uomo Selvaggio. Ma la circolarità del racconto è assicurata 1) dall'aiuto che gli uccelli soccorsi da Gismirante nella fase di andata del suo viaggio gli porgono nella fase del ritorno; 2) dal costante ritornare di Gismirante dalla fata incontrata durante il viaggio di andata; 3) dal fatto che l'avventura col "porco troncascino" ha luogo, guarda caso, proprio nella città natale di Gismirante. Il Gibello è direi, esemplare per quanto riguarda il senso dell'economia nelle sue articolazioni: allontanato in fasce dal suo regno natale e raggiunto il regno della damigella Argogliosa, Gibello abbandona quest'ultimo per andare alla ricerca della famiglia. Durante la quête, s'imbatte nel Cavaliere Nero e nel Cavaliere Vermiglio che lo aiuteranno nella sua prossima operazione di soccorso della principessa, assediata proprio dal padre e dal fratello di Gibello. Fatto prigioniero in Serpentina, e uscito di lì temporaneamente per recar soccorso ad Argogliosa, è nella stessa prigione che la sua lealtà lo riconduce; ma d'altra parte Serpentina fa parte 
dei possedimenti di suo padre, e la stessa duchessa che già lo aveva liberato per permettergli di andare a difendere Argogliosa lo conduce ora alla corte di suo padre. Lì avverranno il riconoscimento, la riconciliazione, e il matrimonio con la principessa opportunamente fatta arrivare.

Bel Gherardino

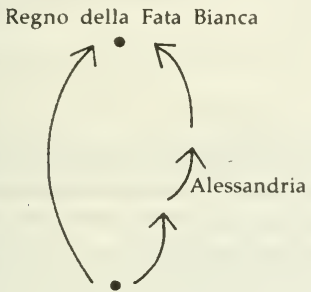

Roma

\section{Liombruno}

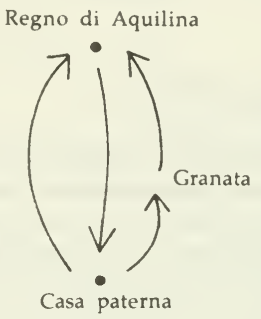

Ponzela Gaia

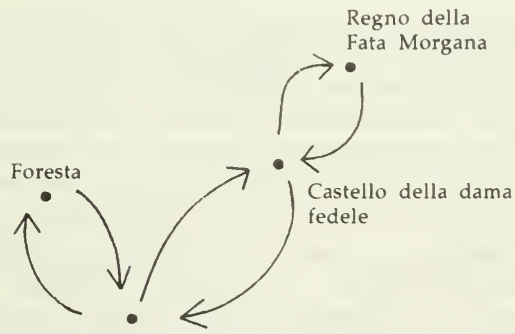

Corte di Artù

Gismirante

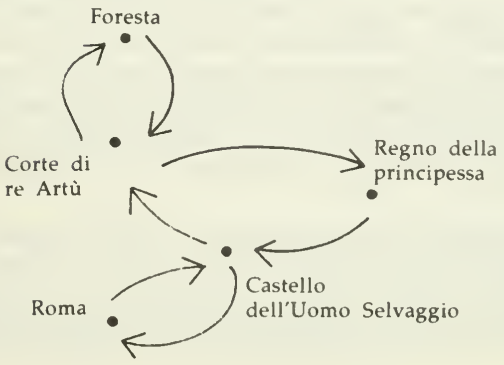


Gibello

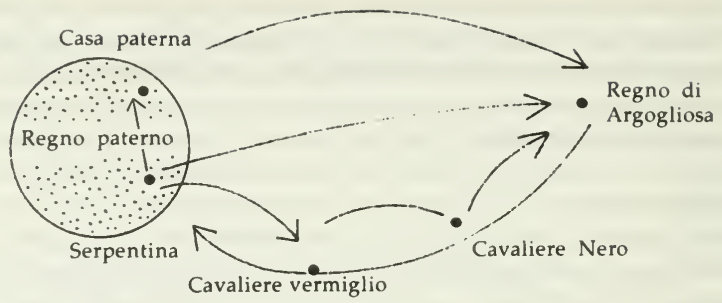

\section{CONCLUSIONI}

Sulla scorta, probabilmente, del Bel Gherardino, a sua volta rifacimento di lais risalenti al Lanval di Marie de France e riduzione del Partonopeus de Blois, assistiamo nella seconda metà del Trecento al consolidarsi di una tradizione narrativa particolare che si appropria la forma del cantare in ottava rima (forma che preesisteva e che si era già realizzata, per esempio, nei Cantari di Tristano o nel poemetto di Fiorio e Biancifiore). Caratteristiche di questa tradizione narrativa sono da riconoscere:

1. Nella presenza di un modello strutturale "ritmico": il pattern narrativo richiede che un protagonista, eventualmente dopo alcune prove di iniziazione, raggiunga un grande bene; ne venga subito dopo fortunosamente privato; intraprenda una quête e riconquisti finalmente il bene perduto. La varie avventure a cui l'eroe va incontro sono unificate da una tensione narrativa orientata, nella prima parte, all'acquisto, nella seconda parte al ri-acquisto del bene perduto. La quête, in particolare, conduce l'eroe in un'alternanza di fortune e di disgrazie ("acquisti" e "perdite"), avanzamenti e retrocessioni nella direzione dell'oggetto della quête, il quale viene però inevitabilmente raggiunto nell 'happy end.

2. Nella brevità, compattezza e "circolarità" dell'architettura del cantare: se vengono seguiti più fili, questi vengono tenuti costantemente presenti e si intersecano lungo il racconto per raggiungere una conclusione comune alla fine del cantare. L'intreccio tende a un massimo di avventure con un minimo di personaggi e di luoghi; è frequente il ritorno dell'eroe al luogo d'origine o a luoghi già visitati; ogni personaggio è normalmente protagonista di diverse azioni.

3. La vicenda vera e propria del cantare è spesso preceduta da una vicenda introduttiva e preparatoria, a cui resta comunque concesso uno spazio molto breve. Il vero e proprio avvio alla vicenda del cantare è dato spesso dalla presenza o dalla "posa" di 
una convenzione sociale (patto, legge, regola di comportamento ecc.), destinata ad essere contraddetta dai fatti.

Per concludere, ci conviene mettere a confronto questo nostro modello con alcuni cantari non compresi nel nostro corpus e vedere se questo resta valido. Tengo però a sottolineare ancora una volta che con l'analisi precedente si è teso a definire una porzione, una determinata varietà della produzione di cantari in ottava rima, fra la fine del ' 300 e i primi del ' 400 , non certo a definire il cantare in generale.

Madonna Lionessa. Del Pucci, un solo cantare di 392 vv.

Madonna Lionessa signoreggia la Lombardia. Suo marito va in Francia a soccorrere il re contro i Saracini e porta infatti un aiuto decisivo; ma si innamora della regina di Francia e, scoperto, è condannato al taglio della lingua. In virtù dei suoi meriti precedenti, ottiene una proroga di cento giorni, e scrive alla moglie avvertendola del pericolo. La moglie allora si traveste e finge di essere Salomone tornato sulla terra per raddrizzare i torti e percorre i paesi amministrando la giustizia. Arriva a Parigi, libera suo marito e se lo porta appresso attraverso varie città d'ltalia. A questo punto si scopre che il marito, nonostante il suo trascorso, ama sua moglie con tutta l'anima e resiste vittoriosamente alle insinuazioni di "Salomone." Salomone si fa riconoscere dal marito e entrambi tornano felici a Milano.

Per quanto Madonna Lionessa non abbia proprio niente di fiabesco e ben poco anche di cavalleresco, vi si può riconoscere lo schema familiare:

partito dalla sua casa, il Capitano raggiunge una posizione invidiabile per il suo soccorso decisivo al re di Francia contro i Saracini. ("acquisto")

Un suo fallo lo precipita nella disgrazia. ("perdita")

Sua moglie, travestita da Salomone, lo libera, (riparazione della "perdita")

ma il Capitano è adesso lontano dalla sua legittima moglie e suo vero amore. Il viaggio insieme a Salomone, che mette alla prova il suo amore per la moglie, costituisce la quête che lo rende degno di riacquistare il bene perduto. (quête e riacquisto del bene perduto)

i(L) - LL - Viol. - Conseq. - AE + iquête - riacquisto del bene perduto'

In questo caso il bene acquistato nella prima parte e quello riacquistato non coincidono. Siamo dunque fuori della sfera d'influenza della "storia di Lanval," ma resiste il modello strutturale.

La Reina d'Oriente. Del Pucci, in quattro cantari. Ma la misura di 4 cantari è anomala, e gli avvenimenti narrati nel 1" e nel $4^{\circ}$ cantare possono facilmente esser riconosciuti come giunte. Ridotta al suo nucleo centrale, anche la storia della Reina d'Oriente si configura in due cantari e si modella sul medesimo schema: 
A causa di un'imprudente anticipazione, la Reina d'Oriente fa allevare la propria figlia come se fosse un maschio. L'imperatore di Roma propone alla corte della Reina d'Oriente il matrimonio della propria figlia con il supposto principe. Non potendo smascherarsi, la fanciulla parte e va a Roma, piena di trepidazione, a celebrare il matrimonio. La notte delle nozze rivela la verità alla sposa, la quale s'acconcia di buon grado a "mantenere verginitade" per la maggior gloria di Dio, e acconsente a coprire l'inganno. Gli "sposi" tornano in oriente e tutto sembra procedere per il meglio. Un'imprudente offesa a una cameriera che era a conoscenza di tutto provoca però il suo desiderio di vendetta, ed essa va a raccontare tutto all'imperatore di Roma. Con una scusa, questi richiama "gli sposi" alla sua corte e, ben deciso a verificare il sesso del genero, fa apprestare una caccia, e poi un bagno. Prevenuta dei disegni dell'imperatore, la figlia della Reina d'Oriente si perde volontariamente nella foresta e vorrebbe uccidersi quando un "cervo-angelo" l'avvisa che ha ricevuto il miracolo: ha infatti cambiato sesso. Tornato alla corte dell'Imperatore, il novello maschio supera brillantemente la prova del bagno, e anche sua moglie può finalmente gustare le dovute gioie matrimoniali.

Istoria di tre giovani disperati e di tre fate. Due cantari. Testimoniato solo da stampe cinquecentine, il cantare riprende però una favola che compare nei Gesta Romanorum (XIV sec.) :

Tre giovani disperati messisi insieme e partiti a cercar fortuna per il mondo, ricevono una sera la visita di tre fate, che fanno un dono a ciascuno di loro : una borsa che offre 100 denari ogni volta che si apre, un tappeto volante, un corno che, a suonarlo, raduna immediatamente 100 cavalieri.

I tre tornano a Roma ma quello cheha ricevuto laborsa si mette in viaggio. In Ispagna, si fa scioccamente derubare della borsa dalla reginotta. Torna allora a Roma e chiede in prestito all'amico il tappeto volante.

Tornato in Ispagna, perde anche quello.

Torna a Roma, e si fa prestare il corno.

Tornato in Ispagna, perde anche quello. (Fine del $1^{\circ}$ cantare).

Per caso mangia dei fichi che gli fanno crescere la coda.

Ancora per caso, mangia dei fichi che gliela fanno scomparire.

Allora torna dalla Reginotta e le fa crescere la coda.

Poi gliene toglie una parte, facendosi restituire gli oggetti magici rubati. ${ }^{27}$

Nel racconto latino, un giovane riceve $i$ tre oggetti magici in eredità dal padre, e la fanciulla che glieli sottrae con l'inganno abita nella stessa città. Nel cantare, i "beni" sono raggiunti in seguito a un'avventura scaturita da una "partenza" dei giovani: immediatamente dopo, i tre tornano a Roma, e di qui il protagonista riparte verso il paese della reginotta, ripetendo la struttura del doppio viaggio che è comune a tutti i cantari del nostro corpus; evidentemente, anche nel far ricorso a una storia conosciuta e definita, l'autore ha dovuto adeguarla alle strutture che il "model$10^{\prime \prime}$ e la tradizione suggerivano.

La varietà di cantari che abbiamo cercato di definire è rimasta viva per un breve periodo di tempo, cedendo ben presto il passo, al livello di letteratura scritta, al lungo poema cavalleresco, mentre le sue storie e i suoi motivi cadevano nel repertorio della "letteratura 
orale ${ }^{\prime \prime}$ popolare. ${ }^{28} \mathrm{Ci}$ è sembrato comunque di non scarso interesse il tentativo di individuare, nella larghissima produzione in ottava rima del primo Rinascimento, una determinata varietà; che può costituire l'avvio a una migliore classificazione e ad una più approfondita conoscenza del cantare italiano.

Université de Montreal

\section{NOTE}

1 Cfr. E. Ragni Cantari nel Dizionario critico della letteratura italiana, vol. I (Torino, 1973), pp. 480-88.

2 P. Zumthor, Essai de poétique médiévale (Parigi, 1972), pp. 339-80.

3 Ediz. a cura di D. De Robertis, "Cantari antichi" in Studi di Filologia italiana, XXVIII (1970), 110-33

4 Questa è la lezione dell'edizione critica a cura di T. Nurmela (Helsinki, 1968). $\dot{E}$ curioso però che la lezione vulgata fino all'edizione critica era "che combatté con l'orsa," mentre nel cantare il sesso dell'animale è ben stabilito dall'incatenamento della rima : orso - soccorso - corso $(1,15)$. Si tratta di un errore meccanico, o della suggestione di una diversa redazione del cantare? Quanto al verbo, se il Boccaccio avesse voluto alludere a una storia conosciuta, effettivamente il passato remoto sarebbe stato più appropriato dell'imperfetto, poiché la lotta con i due mostri è un episodio assolutamente circoscritto. In realtà si tratta di una citazione letterale del cantare, dove dice: "Morto il serpente, e G[herardin] providde/ a Marco Bello, che combattea collo orso/ cridando a voce:-L'orso mi conquide, / se da te, G., non hoe soccorso-" $(I, 15, I-4)$.

5 Ediz. a cura di N. Sapegno in Poeti minori del Trecento (Milano-Napoli, 1952), pp. 84-86.

6 Cfr. G. Varanini, "Sul testo, sull'attribuzione e su una inedita redazione della "storia di Liombruno" in Studi mediolatinie volgari(1954), 250-81.

7 Bel Gher. 1, 10,7 Equando venne in su l'albor del giorno Liombruno. I, v. 201 E quando venne sull'alba del 'giorno Bel Gher. I, 20, 2 Ché chi 'l facesse non potien vedere Liombruno II, v.64 di niuna parte lo potean vedere.

Le coppie di versi si riferiscono nei due cantari a episodi completamente diversi.

8 Ediz. a cura di G. Varanini, in Scelta di curiosità letterarie inedite o rare (Bologna, 1957).

9 La narrazione in prosa della Ghaia Donzella era probabilmente contenuta nelle cc. 65-76 che mancano al manoscritto in questione (Firenze, Naz., II.IV.136).

10 Ediz. a cura di E. Levi, in Fiore di leggende. Cantari leggendari (Bari, 1914), pp. $169-$ 98.

11 E. Levi, Cantari leggendari del popolo italiano (Torino, 1914: GSLI Suppl. n. 16), pp. 95-96.

$12 \mathrm{Mi}$ limito a segnalare la concordanza delle vicende del cantare con quelle di una fiaba russa della celebre raccolta di Afanasiev, Koshceil'immortale, nonché con la fiaba italiana Corpo-senza-l'anima (n.6 della raccolta di Fiabe Italiane di l. Calvino, (Torino, 1956).

13 Ediz. a cura di E. Levi in Fiore di leggende. Cantari leggendari cit., pp. 143-68.

14 G.V. Smithers, "Story-Patterns in some Breton Lays" in Medium Aevum, XXII (1953), 61-92.

15 A. Dundes, The Morphology of North American Indians Folktales (Helsinki, 1964).

16 A. Pasqualino, "Per un'analisi morfologica della letteratura cavalleresca: i'Reali di Francia'," in Uomo e cultura, 5-6 (1970), 76-194.

17 Le sigle sono tratte dalla traduzione francese della seconda edizione dell'opera di Propp: V. Propp, Morphologie du conte (Parigi, 1970). 
18 Fra gli altri schemi elaborati dal Dundes per i miti primitivi troviamo:

\begin{tabular}{rlrl} 
L & \multicolumn{1}{c}{ Dec. } & \multicolumn{1}{c}{ Decpt. } & LL \\
Mancanza & Inganno & Caduta nell'inganno & Riparazione della \\
(= pacte trompeur & (=complicité involontaire & \multicolumn{1}{c}{ mancanza } \\
di Propp) & di Propp)
\end{tabular}

che corrisponde alla vicenda della povertà del pescatore, patto col diavolo, salvataggio di Liombruno; e L - LL che corrisponderebbe alla vicenda di Liombruno abbandonato dal padre e trasportato da Aquilina nel suo regno. La prima parte del Liombruno si strutturerebbe dunque come una successione di schemi mitici. Quanto al Lanval, la distribuzione delle funzioni avverrebbe in maniera differente: L(povertà di Lanval) — LL (amore della fata e ricchezza) Int. (divieto) - Viol. (infrazione del divieto) - Conseq. (conseguenze dell'infrazione) - AE(conseguenze evitate). Nei nostri cantari, ha assunto maggior rilievo l'elemento del viaggio e del ritorno a casa, che li avvicina alla struttura fiabesca.

19 E. Hoeppfner, Les lais de Marie de France (Paris, 1959), p.65.

20 Sul piano dei motivi, si ricorderà che mentre il motivo del cuore custodito successivamente da più animali è un luogo comune fiabesco (si ritrova anche nella favola 156 della raccolta di Afanasiev : Koshicei l'immortale) la caccia al porc blanc è uno degli elementi costitutivi del lai di Guingamor.

21 Una vicenda simile occorre a Partonopeus de Blois, nel poema omonimo.

22 "Infra quel tempo lo misse a studiare/ con un maestro; e da lui bene imprese,/ ed imparò a scrivere e a giostrare,/ e venne in arme prodo uom palese:/ ai suoi colpi nessun potea durare;/ e ben dicea ciascun di quel paese:/-Quest'è figliuol di conte e di barone!-/ tanto era adatto e di bella fazzione." (Liom., 1, 16. Per il v. 3, il Varanini accetta la variante schrimire, al posto di scrivere: cfr. G. Varanini, Sul testo, sull'attribuzione cit., p. 263). Allo stesso modo la principessa Argogliosa, ricevuto Gibello neonato dai mercanti, "tosto lo fece crescere e allevare/ con più maestri a legger e a studiare./ Egli era veramente tanto destro,/ il gaio giovinetto, ad ogni cosa,/ che da ciascuno era tenuto maestro/ e la sua fama cresce valorosa./ Ed alle cose era maniero e presto/ vie $\mathrm{me}^{\prime}$ che gli altri. . . Crescendo il giovinetto valoroso/ alla schermaglia cominciò a usare,/ della qual venne tanto copioso/ che a quel paese non trovava pare. Di costumi /e di danze più gioioso/ più che null'altro me' le sapea fare,/ di salti e di lanciare e di destrezza, / e in belle cacce tuttora s' avvezza" (Gibello, 11, 7-8; 12, 1-6; 13, 1-8).

23 Così fu.G[herardin] suo servidore,/ che di tale arte era molto sottile;/ e quel signore gli puose molto amore,/ che quasi tutti gli altri tenne a vile./ E la reina ne 'nfiammò nel core,/ perché el[1]a il vedea cotanto gentile "(Bel Gher. 11, 21, $1-6)$.

$24 \mathrm{Ci}$ soccorre in questa fase dello studio l'analisi di A. Pasqualino sui Reali di Francia di Andrea da Barberino che, nelle intenzioni stesse dell'autore vuole essere un contributo alla definizione morfologica del romanzo cavalleresco: A. Pasqualino, Per un'analisi morfologica della letteratura cavalleresca: $i$ "Reali di Francia" cit.

25 E. Dorfman, The Narreme in the Medieval Romance Epic. An Introduction to Narrative Structures (Toronto, 1969).

26 Sul piano semiologico una simile caratteristica implica naturalmente la coscienza di appartenere a un mondo diverso e un giudizio (negativo) di autori e pubblico sugli istituti storici rappresentati. Non sarà casuale che l'unico cantare penetrato tanto profondamente nella coscienza popolare da essere ancora vivo nel folclore odierno sia il Liombruno, che appare più libero da riferimenti storici, e che sembra trarre i motivi del suo racconto da un humus allo stesso tempo più profondo e più semplice.

27 Ediz. a cura di A. Keller. (Stuttgart, 1842), cap. CXX: De amicitiae verae probatione (Cfr. E. Levi I cantarileggendari cit., p. 58).

28 Si vedano le novelle nn. 6, 46, 48, 55, 66, 69, 189 delle Fiabe italiane raccolte e trascritte da I. Calvino. 